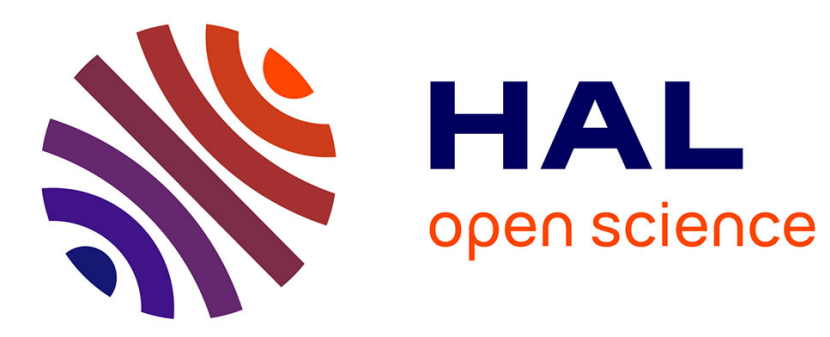

\title{
Advances in quantum optical frequency combs
}

Thomas Daugey, Amélie Piveteau, Souleymane Diallo, Kouomou Yanne

Chembo, Jean Merolla

\section{To cite this version:}

Thomas Daugey, Amélie Piveteau, Souleymane Diallo, Kouomou Yanne Chembo, Jean Merolla. Advances in quantum optical frequency combs. SPIE LASE, Feb 2019, San francisco, United States. 10.1117/12.2510444 . hal-02382651

\section{HAL Id: hal-02382651 \\ https://hal.science/hal-02382651}

Submitted on 7 Dec 2020

HAL is a multi-disciplinary open access archive for the deposit and dissemination of scientific research documents, whether they are published or not. The documents may come from teaching and research institutions in France or abroad, or from public or private research centers.
L'archive ouverte pluridisciplinaire HAL, est destinée au dépôt et à la diffusion de documents scientifiques de niveau recherche, publiés ou non, émanant des établissements d'enseignement et de recherche français ou étrangers, des laboratoires publics ou privés. 


\title{
Advances in quantum optical frequency combs
}

\author{
T. Daugey ${ }^{1}$, A. Piveteau ${ }^{1}$, S. Diallo ${ }^{1}$, Y. K. Chembo ${ }^{1,2, *}$ and J.-M. Merolla ${ }^{1}$ \\ ${ }^{1}$ FEMTO-ST Institute, Univ. Bourgogne Franche-Comté, CNRS, \\ 15B Avenue des Montboucons, 25030 Besançon cedex, France \\ ${ }^{2}$ Now at the Institute for Research in Electronics and Applied Physics (IREAP), and the \\ Department of Electrical and Computer Engineering, University of Maryland, College Park \\ MD 20742, USA. \\ ${ }^{*}$ Corresponding author
}

\begin{abstract}
The quantum properties of optical frequency combs have been the focus of several research works in recent years. Investigating the quantum correlations between the spectral components of the combs is of fundamental interest because it allows for a better understanding of light-matter interactions, but also of technological interest as it wold permits the implementation of quantum communication networks. In this communication, we present some of our latest advances in this field.
\end{abstract}

Keywords: optical frequency combs, quantum correlations

\section{INTRODUCTION}

In recent years, optical frequency combs have been mostly investigated for the purpose of time-frequency metrology and sensing.

Several research groups have studied in detail the frequency combs generated by nonlinear whispering gallery mode (WGM) with ultra-high $Q$ factors, when pumped by a continuous-wave laser. ${ }^{1-3}$ The nonlinear analysis of these combs can be performed via a modal expansion approach ${ }^{4,5}$ or via a spatiotemporal formalism ${ }^{6-8}$ relying on the Lugiato-Lefever equation. ${ }^{9}$ For the particular case of Kerr combs in the quantum regime, ${ }^{10}$ sub-threshold pumping leads to twin-photon generation leading to parametric fluorescence, ${ }^{11-25}$ while pumping above threshold can lead to squeezing, ${ }^{26}$ as initially predicted by several decaes ago for systems ruled by the Lugiato-Lefever equation. ${ }^{27}$ Indeed, other types of nonlinearities can be used as well for the generation of quantum states using high-Q resonators ${ }^{28-30}$ Such quantum optical frequency combs are expected to play a major role in future
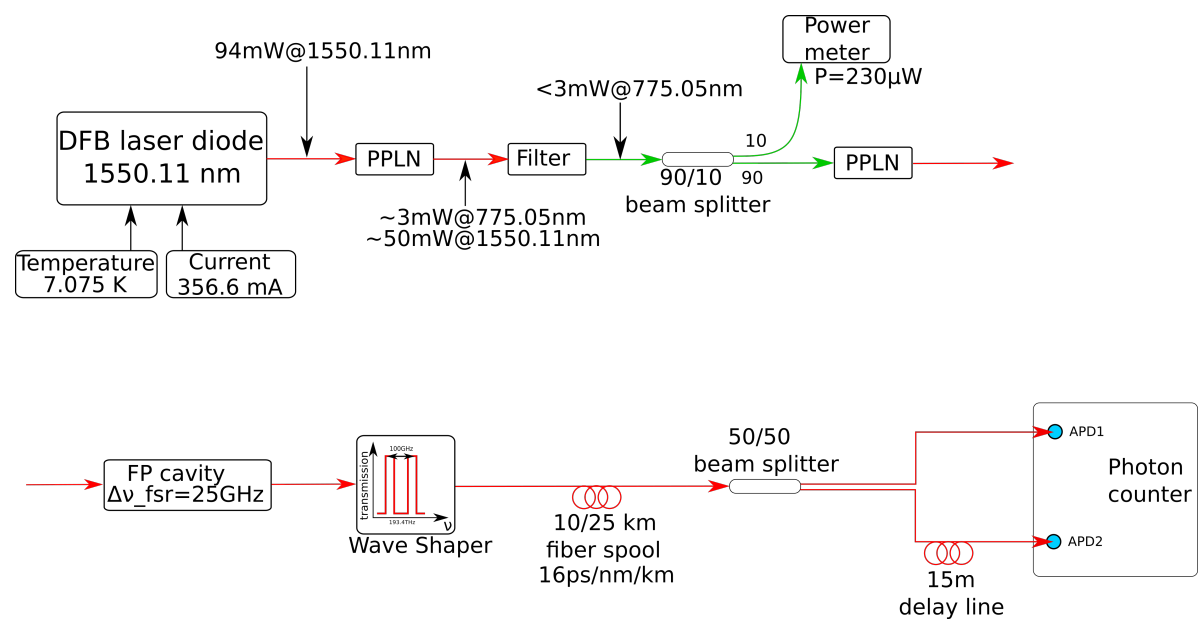

Figure 1. Experimental setup for photon pair generation and frequency comb filtering. The superior block is the source and the inferior block highlights photon manipulation, that is filtering, delay and detection 


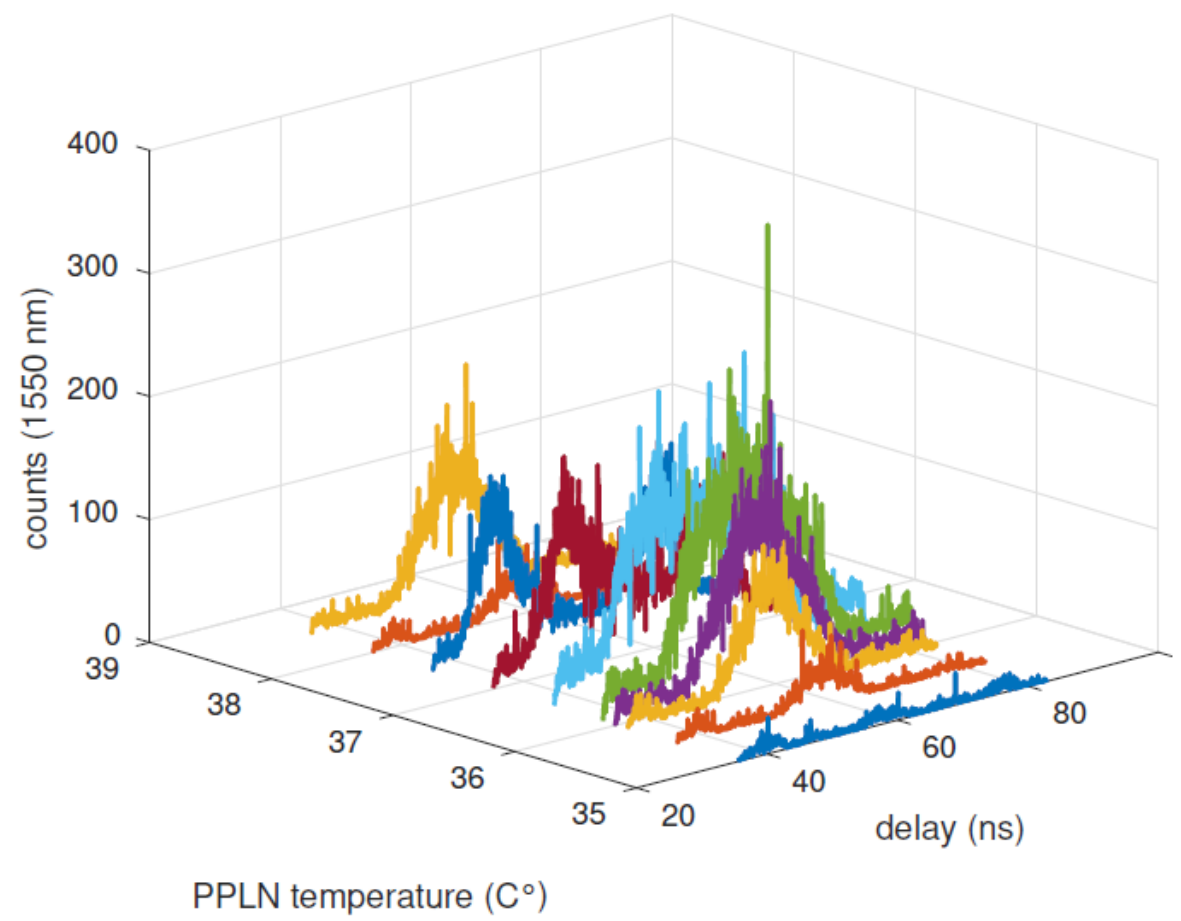

Figure 2. Spontaneous emission of the PPLN at $1550.11 \mathrm{~nm}$ mesured with $10 \mathrm{~km}$ fiber spool and the photon counter.

quantum communication networks. The topic of optical frequency comb generation using high- $Q$ WGM of ring resonators has generated an extensive literature in both the nonlinear and quantum regimes, and comprehensive reviews include for example refs. ${ }^{31-34}$

In this communication we present preliminary results related to our work that is based on a different kind of frequency optical frequency comb, and the main idea here is to carve out a comb out of a fluorescence spectrum generated via spontaneous parametric down conversion, and the system presented below is essentially an experimental characterization of our twin photon source.

\section{SYSTEM}

The experimental system under study is presented in Fig. 1. An important procedure is the measurement of the coincidence detection for two photons, that allows us to find out, with a given probability, if those two photons belong to the same pair. We use very sensitive avalanche photodiodes that can perform single-photon detection, and the performance of our measurement procedure critically depends on various parameters, such as the dark count rate. Our photon counters are used in the triggered mode and we measure the time between the two triggers in order to plot coincidence histograms over a certain period of time.

The photon pairs are generated not by four wave mixing as in a sub-threshold Kerr comb generator, but via spontaneous parametric down conversion inside a PPLN crystal. A fibered Fabry-Perot cavity filters the spontaneous emission spectrum of the PPLN to give us the equivalent of a frequency comb.

The source block is composed of a laser diode that with an output around $1550 \mathrm{~nm}$. The laser diode pumps the PPLN crystal and part of the the output signal is the second harmonic generation (SHG) around $775 \mathrm{~nm}$. The power generated through second harmonic generation is used to pump another PLLN crystal, this time to generate spontaneous emitted photon pairs. Since at the output of the first PPLN there is remaining at $1550 \mathrm{~nm}$, a broadband filter is used to filter out around the $1550 \mathrm{~nm}$ signal and let the photons at $775 \mathrm{~nm}$ pass. A 90/10 coupler is used to send a small fraction the light to a power meter, which allows us to monitor the input power and perform power stability measurements. A fibered Fabry-Perot cavity with a $25 \mathrm{GHz}$ free spectral range is 


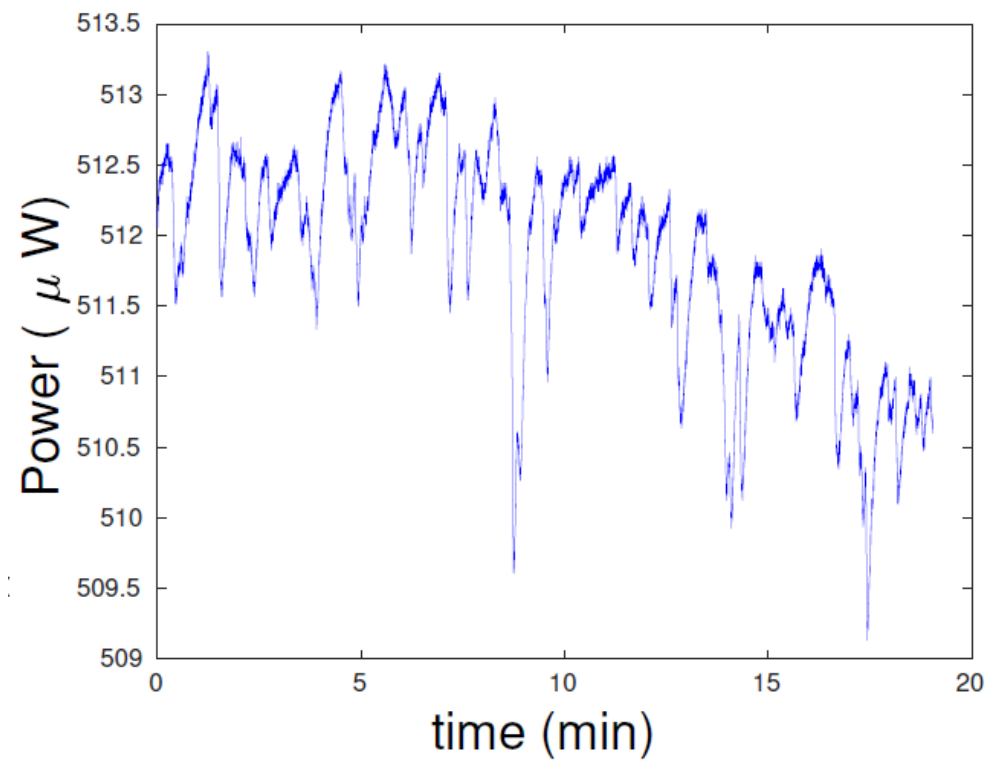

Figure 3. Power measurement of the output power of the first PPLN (775.05 nm) over 20 minutes

used to periodically filter the spontaneous emission spectrum of the second PPLN. A programmable filter is then used to select resonances of the Fabry-Perot cavity and thus frequency of the photon pairs that are generated. A fiber spool of varying length (10 or $25 \mathrm{~km}$ ) is used to temporally delay the photons relative to their frequency. The fiber turns a frequency difference into a time of arrival difference because of its dispersion. For silica fiber, the group velocity dispersion is typically $16 \mathrm{ps} / \mathrm{nm} / \mathrm{km}$ (i.e., two photons separated by $1 \mathrm{~nm}$ and entering the fiber at the same time are going to exit with a time delay of $16 \mathrm{ps}$ after $1 \mathrm{~km}$ of fiber propagation). Finally a beam splitter separates the photon pairs and send one to the first detector and the other to the second detector.

\section{RESULTS AND OUTLOOK}

Figure 3 represents the count histogram for different temperatures of the PPLN. We started at $35^{\circ} \mathrm{C}$ and finished at $38.5^{\circ} \mathrm{C}$ and for each temperature, the counts were measured over 20 minutes. We found that the ideal temperature, that the temperature for which the number of counts is maximized was $36.8^{\circ} \mathrm{C}$. From that point on, this is the temperature we used.

On the other hand, in Fig. 3, the power is very stable over 20 minutes with relative variations of less that $1 \%$. Over 2 hours we observed variations of about 5 to $10 \%$ but since most of our measurements where made over 20 minutes, the first stability measurement is thus more relevant.

The next step of our investigations will be to optimize the system in order to increase brightness and stability over time. A detailed analysis will be performed as well to study its quantum correlation properties. ${ }^{44-47}$ Future work will also be specifically devoted to the use of high-Q WGM resonators in the linear (filtering) or nonlinear (twin-photon generation) regime for various quantum photonic systems. ${ }^{35-43}$

\section{REFERENCES}

1. T. J. Kippenberg, S. M. Spillane, and K. J. Vahala, Kerr-Nonlinearity Optical Parametric Oscillation in an Ultrahigh-Q Toroid Microcavity, Phys. Rev. Lett. 93, 083904 (2004).

2. A. A. Savchenkov, A. B. Matsko, D. Strekalov, M. Mohageg, V. S. Ilchenko, and L. Maleki, Low Threshold Optical Oscillations in a Whispering Gallery Mode CaF 2 Resonator, Phys. Rev. Lett. 93, 243905 (2004).

3. P. Del'Haye, A. Schliesser, A. Arcizet, R. Holzwarth, and T. J. Kippenberg, Optical frequency comb generation from a monolithic microresonator, Nature 450, 1214 (2007). 
4. Y. K. Chembo and N. Yu, Modal expansion approach to optical-frequency-comb generation with monolithic whispering-gallery-mode resonators, Phys. Rev. A 82, 033801 (2010).

5. Y. K. Chembo and N. Yu, On the generation of octave-spanning optical frequency combs using monolithic whispering-gallery-mode microresonators, Opt. Lett. 35, 2696 (2010).

6. A. B. Matsko, A. A. Savchenkov, W. Liang, V. S. Ilchenko, D. Seidel, and L. Maleki, Mode-locked Kerr frequency combs, Opt. Lett. 36, 2845 (2011).

7. Y. K. Chembo and C. R. Menyuk, Spatiotemporal Lugiato-Lefever formalism for Kerr-comb generation in whispering-gallery-mode resonators, Phys. Rev. A 87, 053852 (2013).

8. S. Coen, H. G. Randle, T. Sylvestre, and M. Erkintalo, Modeling of octave-spanning Kerr frequency combs using a generalized mean-field Lugiato-Lefever model, Opt. Lett. 38, 37 (2013).

9. L. A. Lugiato and R. Lefever, Spatial Dissipative Structures in Passive Optical Systems, Phys. Rev. Lett. 58, 2209 (1987).

10. Y. K. Chembo, Quantum dynamics of Kerr optical frequency combs below and above threshold: spontaneous four-wave mixing, entanglement, and squeezed states of light, Phys. Rev. A 93, 033820 (2016).

11. J. E. Sharping, K. F. Lee, M. A. Foster, A. C. Turner, B. S. Schmidt, M. Lipson, A. L. Gaeta and Prem Kumar, Generation of correlated photons in nanoscale silicon waveguides, Optics Express 14, 12388 (2006).

12. S. Clemmen, K. Phan Huy, W. Bogaerts, R. G. Baets, Ph. Emplit, and S. Massar, Continuous wave photon pair generation in silicon-on-insulator waveguides and ring resonators, Optics Express 17, 16558 (2009).

13. L. G. Helt, Z. Yang, M. Liscidini, and J. E. Sipe, Spontaneous four-wave mixing in microring resonators, Opt. Lett. 35, 3006 (2010).

14. J. Chen, Z. H. Levine, J. Fan, and A. L. Migdall, Frequency-bin entangled comb of photon pairs from a Silicon-on-Insulator micro-resonator, Optics Express 19, 1470 (2011).

15. S. Azzini, D. Grassani, M. J. Strain, M. Sorel, L. G. Helt, J. E. Sipe, M. Liscidini, M. Galli, and D. Bajoni, Ultra-low power generation of twin photons in a compact silicon ring resonator, Optics Express 20, 23100 (2012).

16. L. G. Helt, M. Liscidini, and J. E. Sipe, How does it scale? Comparing quantum and classical nonlinear optical processes in integrated devices, J. Opt. Soc. Am. 29, 2199 (2012).

17. S. Azzini, D. Grassani, M. Galli, L. C. Andreani, M. Sorel, M. J. Strain, L. G. Helt, J. E. Sipe, M. Liscidini, and D. Bajoni, From classical four-wave mixing to parametric fluorescence in silicon microring resonators, Optics Express 37, 3807 (2012).

18. R. M. Camacho, Entangled photon generation using four-wave mixing in azimuthally symmetric microresonators, Optics Express 20, 21977 (2012).

19. N. Matsuda, H. Le Jeannic, H. Fukuda, T. Tsuchizawa, W. J. Munro, K. Shimizu, K. Yamada, Y. Tokura and $\mathrm{H}$. Takesue, A monolithically integrated polarization entangled photon pair source on a silicon chip, Sci. Rep. 2, 817 (2012).

20. C. Reimer, L. Caspani, M. Clerici, M. Ferrera, M. Kues, M. Peccianti, A. Pasquazi, L. Razzari, B. E. Little, S. T. Chu, D. J. Moss, and R. Morandotti, Integrated frequency comb source of heralded single photons, Optics Express 22, 6535 (2014).

21. Z. Vernon and J.E. Sipe, Spontaneous four-wave mixing in lossy microring resonators, Phys. Rev. A 91, 053802 (2015).

22. E. Engin, D. Bonneau, C. M. Natarajan, A. S. Clark, M. G. Tanner, R. H. Hadfield, Sanders N. Dorenbos, V. Zwiller, K. Ohira, N. Suzuki, H. Yoshida, N. Iizuka, M. Ezaki, J. L. O'Brien, and M. G. Thompson, Photon pair generation in a silicon micro-ring resonator with reverse bias enhancement, Optics Express 21, 27826 (2013).

23. D. Grassani, S. Azzini, M. Liscidini, M. Galli, M. J. Strain, M. Sorel, J. E. Sipe, and D. Bajoni, Micrometerscale integrated silicon source of time-energy entangled photons, Optica 2, 88 (2015).

24. J. W. Silverstone, R. Santagati, D. Bonneau, M. J. Strain, M. Sorel, J. L. O'Brien and M. G. Thompson Qubit entanglement between ring-resonator photon-pair sources on a silicon chip, Nature Commun. 6, 7948 (2015).

25. R. Wakabayashi, M. Fujiwara, K.-I Yoshino, Y. Nambu, M. Sasaki, T. Aoki, Time-bin entangled photon pair generation from Si micro-ring resonator, arXiv:1501.05687 [quant-ph] (2015). 
26. A. Dutt, K. Luke, S. Manipatruni, A. L. Gaeta, P. Nussenzveig, and M. Lipson, On-Chip Optical Squeezing, Phys. Rev. Applied 3, 044005 (2015).

27. L. A. Lugiato and F. Castelli, Quantum Noise Reduction in a Spatial Dissipative Structure, Phys. Rev. Lett. 68, 3284 (1992).

28. J. U. Fürst, D. V. Strekalov, D. Elser, A. Aiello, U. L. Andersen, Ch. Marquardt, and G. Leuchs, Quantum Light from a Whispering-Gallery-Mode Disk Resonator, Phys. Rev. Lett. 106, 113901 (2011).

29. M. Förtsch et al., A versatile source of single photons for quantum information processing, Nature Communications 4, 1818 (2013).

30. M. Förtsch, G. Schunk, J. U. Frst, D. Strekalov, T. Gerrits, M. J. Stevens, F. Sedlmeir, H. G. L. Schwefel, S. W. Nam, G. Leuchs, and Ch. Marquardt, Highly efficient generation of single-mode photon pairs from a crystalline whispering-gallery-mode resonator source, Phys. Rev. A 91, 023812 (2015).

31. Y. K. Chembo, Kerr optical frequency combs: theory, applications and perspectives, Nanophotonics 5, 214 (2016).

32. D. V. Strekalov, C. Marquardt, A. B. Matsko, H. G. Schwefel, and G. Leuchs, Nonlinear and quantum optics with whispering gallery resonators, J. Opt. 18, 123002 (2016).

33. G. Lin, A. Coillet, and Y. K. Chembo, Nonlinear photonics with high-Q whispering-gallery-mode resonators, Adv. Opt. Phot. 9828 (2017).

34. A. Pasquazi, M. Peccianti, L. Razzari, D. J. Moss, S. Coen, M. Erkintalo, Y. K. Chembo, T. Hansson, S. Wabnitz, P. Del'Haye, X. Xue, A. M. Weiner, and R. Morandotti, Micro-combs: a novel generation of optical sources, Phys. Rep. 729, 1 (2018).

35. H. Tavernier, P. Salzenstein, K. Volyanskiy, Y. K. Chembo, L. Larger, Magnesium fluoride whispering gallery mode disk-resonators for microwave photonics applications, IEEE Phot. Technol. Lett. 22, 1629 (2010).

36. A. Coillet, R. Henriet, K. P. Huy, M. Jacquot, L. Furfaro, I. Balakireva, L. Larger, and Y. K. Chembo, Microwave Photonics Systems Based on Whispering-gallery-mode Resonators, J. Vis. Exp. 78, e50423 (2013).

37. G. Lin, S. Diallo, K. Saleh, R. Martinenghi, J.-C. Beugnot, T. Sylvestre, and Y. K. Chembo, Cascaded Brillouin lasing in monolithic barium fluoride whispering gallery mode resonators, Appl. Phys. Lett. 105, 231103 (2014).

38. G. Lin and Y. K. Chembo, On the dispersion management of fluorite whispering-gallery mode resonators for Kerr optical frequency comb generation in the telecom and mid-infrared range, Opt. Express 23, 1594 (2015).

39. Y. K. Chembo, I. S. Grudinin and N. Yu, Spatiotemporal dynamics of Kerr-Raman optical frequency combs, Phys. Rev. A 92, 043818 (2015).

40. R. Henriet, G. Lin, A. Coillet, M. Jacquot, L. Furfaro, L. Larger, and Y. K. Chembo, Kerr optical frequency comb generation in strontium fluoride whispering-gallery mode resonators with billion quality factor, Opt. Lett. 40, 1567 (2015).

41. G. Lin and Y. K. Chembo, Phase-locking transition in Raman combs generated with whispering gallery mode resonators, Opt. Lett. 41, 3718 (2016).

42. G. Lin, S. Diallo, J. M. Dudley and Y. K. Chembo, Universal nonlinear scattering in ultra-high Q whispering gallery-mode resonators Opt. Express 24 (14880 (2016).

43. K. Saleh and Y. K. Chembo, On the phase noise performance of microwave and millimeter-wave signals generated with versatile Kerr optical frequency combs, Opt. Express 2425043 (2016).

44. M. Bloch, S. W. McLaughlin, J.-M. Merolla, and F. Patois, Opt. Lett. 32, 301-303 (2007).

45. L. Olislager, J. Cussey, A. T. Nguyen, P. Emplit, S. Massar, J.-M. Merolla, and K. P. Huy, Phys. Rev. A 82, $013804(2010)$.

46. L. Olislager, I. Mbodji, E. Woodhead, J. Cussey, L. Furfaro, P. Emplit, S. Massar, K. P. Huy, and J.-M. Merolla, New Journal of Physics 14, 043015 (2012).

47. L. Olislager, E. Woodhead, K. P. Huy, J.-M. Merolla, P. Emplit, and S. Massar, Phys. Rev. A 89, 052323 (2014). 